\title{
Women's Sexual Experiences and Adjustment after Cervical Cancer Treatment in Tikur Anbessa Specialized Hospital, Addis Ababa- Ethiopia: A Qualitative Study
}

Teshome Habte ( $\nabla$ teshome.habte@aau.edu.et )

Addis Ababa University

Gashaw Yada

Addis Ababa University

Endalew Gemechu

Addis Ababa University

\section{Research Article}

Keywords: Cervical cancer, sexual health, sexual experience, adjustment, quality of life

Posted Date: March 24th, 2021

DOl: https://doi.org/10.21203/rs.3.rs-313724/v1

License: (c) (i) This work is licensed under a Creative Commons Attribution 4.0 International License.

Read Full License 


\section{Abstract}

Background: Cervical cancer is one of the most common cancers and the leading causes of cancer deaths globally.

Objective: This study aimed to explore the experiences of women in sexual adjustment after treatment of cervical cancer at Tikur Anbesa Specialized hospital.

Method: A descriptive phenomenological qualitative approach and semi-structured in-depth interview method were used and 14 clients recruited to collect data from 2nd February to 15th March 2019. The interviews were audio-taped and analyzed using the procedures of thematic content qualitative data analysis.

Results: In this finding, four themes emerged for data analysis, namely, Treatment Side effect, Sexual Problems after radiation therapy, Knowledge deficit, and poor coping strategies of sexual relationships which reflect women with cervical cancer treatment problems.

Conclusion and Recommendations: This finding reveals that the Sexual life of women affected by physical changes brought by cancer therapy, information gaps, or knowledge deficit related to cervical cancer and pelvic radiotherapy. the researcher recommended that there should be broad promotion including counseling service and integrated service set up to alleviate sexual related problems and treatment side effect for cervical cancer.

\section{Introduction}

\subsection{Background}

The most common female cancer in the world with an estimated incidence and mortality of 528,000 and 266,000 respectively is cervical cancer (CC)[1]. Women with cervical cancer experience several types of physical changes on the vaginal anatomical structure and size as reported by research studies. These changes can affect women body image distortion and conflict with their partner [2]. Post-treatment disruptions to sexual functioning, such as reduced libido and dyspareunia, commonly persist when other areas, such as mental health and social adjustment, have normalized [3].

According to global cancer incidence, mortality and prevalence, there were about 7,095 newly diagnosed cervical cancer cases and 4,732 cervical cancer deaths in 2012 in Ethiopia, accounting for about $17.0 \%$ of total cancer cases and deaths in females. Cervical cancer ranks as the second most frequent cancer among women in Ethiopia [4], there is an increasing number of long-term survivors for whom quality of life is major importance in spite of lack of rehabilitation support based on their need and interest thus, this study aimed at providing in-depth information of cervical cancer patient sexual experience and sexual adjustment for better understanding of the experiences of this specific group could inform the provision of supportive care services in Tikur Anbessa Specialized Hospital. 


\subsection{Statement of the problem}

Currently, cervical cancer in the developing nations is heavily falling with the magnitude of $85 \%$ of the cases and $88 \%$ of the deaths due to cervical cancer. The lifetime risk and problem associated with cervical cancer in developing countries is $35 \%$ with greater lifetime risk than women in high income countries. In Ethiopia, where oncology practice is so young and also, awareness among health professionals about oncology is very low. Most African countries including Ethiopia have no standard a cancer registry. The Ethiopian oncology service was started in Tikur Anbessa specialized hospital in the 1998 Ethiopian calendar.

The growing population in Ethiopia coupled with lifestyle changes will lead to an increasing burden of cancer despite oncology services being wholly inadequate, no cancer registry exists, and only one cancer treatment center, with a few doctors and nurses struggling to serve the entire country [5]. Cancer treatment significantly affects the sexual function of the patients. Providing and discussing information regarding sexuality problems with the patients are some important measures that should be done by the healthcare professionals $[6,7]$. The sexual behavior of women needs to be addressed holistically because sexuality is a comprehensive construct that requires the incorporation of a variety of individual, social and cultural dimensions [8].

\subsection{Significance of the study}

This study is capable of advocating a broad view of sexuality, emphasizing relationship and sensuality as essential components for sexual satisfaction. It makes an effort to uncover trends in thought and opinions, and dive deeper into the problem, and has the potential to generate answers to complex questions. Understanding the influence of cervical cancer on survival is crucial to enable health and social professionals to strengthen strategies to enhance the quality of life of women surviving cervical cancer. The findings of this study also serve as a baseline information for other large-scale studies and may help for health care providers in actual practices and to improve woman's sexual health and quality of life. It is also providing an immediate solution to the problem or helps to develop ideas or hypotheses for potential quantitative research. Moreover, the study can be an input to policymakers, program managers, health professionals at various levels of health institutions for evidence-based planning and interventions.

\section{Research Questions/hypothesis}

1. What are the sexual experiences of women in post cervical cancer treatment?

2. What are coping- strategies (adjustments) for the treatment side-effects of the treatment of women with cervical cancer?

\section{Purpose Of The Study}


This study designed to explore the experiences of women in sexual adjustment after treatment of cervical cancer at Tikur Anbesa Specialized Hospital.

\section{Research Method}

\section{- Study Site and Time Frame}

This study was conducted at Tikur Anbessa Specialized Hospital located in Addis Ababa city administration. It is a teaching, central tertiary generalized referral hospital with approximately 800 inpatients beds [9]. The oncology unit has four oncologists, one hematologist, three radiotherapists, and 15 nurses working in the unit. This oncology unit is providing comprehensive service which includes: cancer registry, radiotherapy, chemotherapy, and palliative care.

This study conducted from February to May 2019.

\section{- Study Participants}

Data were collected from patients with cervical cancer who were completed their clinical diagnose and treatments three months before the study period. Initially, the investigator planned to interview 8 patients but the number of participants has been increased to 13 due to the fact that data acquired from the interview was continued to vary and the researcher wanted to gather adequate and insightful information regarding women's sexual experiences and their coping methods after treatment of cervical cancer.

\section{- Study design}

A qualitative study design was employed for this study. The rationale behind using the qualitative research method was qualitative research is well suited for understanding phenomena in their context, exploring their feeling, opinion, suggestions, concepts, and behaviors [10]. A qualitative method is appropriate for this study because the aim is to realize and describe human experiences like women's sexual experience after cervical cancer treatment and its coping strategies. And human emotions or experiences are not easy to quantify or assign numerically. therefore, a qualitative approach is appropriate. Evidence from more than one case is often considered to be stronger than evidence from a single case. [11].

\section{- Sampling Technique and Procedure}

A purposive sampling technique were used as it is essential to identify and include information rich cases that could give a full understanding of the women's sexual experience and their coping strategies after cervical cancer treatment.

\section{- Inclusion and Exclusion criteria:}


Inclusion criteria: women patients over the age of eighteen years, treated as cervical cancer patients at TASH for more than three months are inclusion criteria.

Exclusion criteria: for the study were comprised of women who were not sexually active and received less than the 3-month course of cervical cancer treatment, mentally ill patients are excluded from the study.

\section{- Definition of Terms and Concepts}

- Women patients: women with cervical cancer who were treated as cervical cancer and under medical care follow-up and treated and age greater than eighteen.

- Cervical Cancer: Cervical cancer is generally defined as a disease characterized by the abnormal growth of cells in the cervix, the region of the uterus that joins the vagina. Dorland's illustrated medical dictionary (1994) describes cervical cancer as an infection in which the cells of the cervix become abnormal and start to grow uncontrollably, forming tumors and a common cause of cancer deaths in women. In light of this study, cervical cancer will be considered as those abnormal growths of cells of the cervix, which necessitate treatment.

- Experience: This term is self-exploratory. The oxford concise dictionary (1997) defines

experiences as actual observation of practical links with the facts and events and which leaves a lasting impression. This, therefore, suggests views and perceptions of patients.

- Sexual experience: is the past events, knowledge, practice, and feelings in sexual activity

- Sexuality: is a person's capacity for sexual feelings.

- Adjustment: is defined as a process wherein women after cervical treatment builds

variations in the behavior to achieve harmony with her, aim to maintain the state of equilibrium between her and her sexual experience.

\section{- Data Collection Technique and Analysis Procedures}

In-depth interviews were employed for this study. The researcher used in-depth interviews as a primary tool for data collection. The interview is an essential source of information. Data analysis was done simultaneously with data collection since the design of the research is qualitative. To do this, an in-depth interview was recorded and written down in the paper and then translated into English. Furthermore, during the interview field notes were taken concerning the participant's gestures, tones, and other body languages. In doing so, the researcher followed the thematic content analysis method which involves transcription, translation, coding, and categorization, and develops themes and interpretations the researcher intended to do it manually. 
The thematic analysis focuses on the coding of quality data, producing clusters of text with similar meaning often searching for the fundamental them and capturing the real meaning of the phenomena under exploration [12].

\section{- Measures taken to Improve Trustworthiness of the Data}

Trustworthiness is the ability of researchers to convince participants and themselves that the findings of the inquiry are direct, truthful, or reliable [13]. Qualitative research is trustworthy when it accurately represents the experience of the study participants [14]. Trustworthiness is enhanced (improved or increased in quality) by credibility, transferability, and conformability [15]. Credibility refers to "the confidence in the truth of the data". The true value of this study was obtained from the sexual experience of cervical cancer patients after treatment, the research design used, the method of data collection, and the context in which the study was conducted [16]. The researcher has applied three techniques to achieve credibility: My experience with cancer issues and terminologies which the researcher was developed during engagement in field practicum in the institution and in other health-related courses, triangulation of data from different sources (secondary and primary sources), and allowed peer checking of the final result. Transferability refers to "the extent to which the findings can be applied in other contexts or with other participants". As the research is qualitative by its nature, the researcher was not interested in the generalization of the findings. All data were defined in terms of the specific context in which they occurred. Conformability refers to "the degree to which the findings are the product of the focus of the inquiry and not the biases of the researcher". A conformability audit trial must ensure that conclusions, interpretations recommendations can be traced to their sources and if they are supported by the inquiry [17]. The following ensure conformability of data when the interview is conducted, Field notes were taken furthermore the interviews were tape-recorded hence the raw data is available. By refining the data collection instrument in the course, by using codes and categorization, and by developing themes from the coded data were analyzed without my personal biases.

\section{Results}

\subsection{Participant's Socio-Demographic characteristics}

Thirteen women fulfilled the eligibility criteria plus one gynecologist doctor participated a total of fourteen participants included in this study. In general, among fourteen women undertaken face to face in-depth interviews. All participants within the age range of (19-65). All received radiation treatment from these eight women received external beam radiation treatment, two received combined treatment of external beam radiation treatment and surgery, and the rest three received external beam radiation with Brachytherapy for their cervical cancer disease treatment.

\subsection{Emerged themes}

From the analysis of individual interview data, four themes emerged as depicted table 1 below. These themes were identified as the rich and detailed account of the experiences of sexually active women who 
have received at least a 3-month course of pelvic radiotherapy for cervical cancer at Tikur Anbessa Specialized Hospital.

Table 1: four emerged themes used for individual data analysis of pelvic radiotherapy for cervical cancer at Tikur Anbessa Specialized Hospital.

\begin{tabular}{|lll|}
\hline No & Theme & Categories \\
\hline 1 & Treatment Side effect & - Immediate effect \\
& & - Late effect \\
\hline 2 & $\begin{array}{l}\text { Sexual Problems after radiation } \\
\text { therapy }\end{array}$ & - Sexual unwillingness \\
& - Fear of sexual intercourse due to excessive pain \\
\hline 3 & Knowledge deficient & $\begin{array}{l}\text { - Inadequate information received from the health } \\
\text { professionals }\end{array}$ \\
\hline 4 & $\begin{array}{l}\text { Coping strategies of sexual } \\
\text { relation }\end{array}$ & - Husband waited for treatment process \\
& & - Not feeling like a woman \\
& & - Stopped having sexual relation for ever \\
\hline
\end{tabular}

\subsection{Treatment Side effect}

All women received radiation treatment for their cervical cancer care. Eight women received external beam radiation treatment, two received combined treatment of external beam radiation treatment and surgery, and the rest three received external beam radiation with Brachytherapy for their cervical cancer disease treatment. Most women who received radiotherapy reported various treatment side effects immediately and in the course of treatment.

\subsection{Unwanted immediate treatment effect}

Women after cervical cancer treatment, according to their report they developed diarrhea, itching, headache, heartburn, vomiting, poor appetite, pain, skin change in texture and color, burning sensation while urinate and Vaginal discharge. The main reason combine treatment like physiologic and psychosocial treatment is due to prolonged treatment period. In addition to that as they reported as symptoms were subsiding without medical intervention, but some of the symptoms last long like burning sensation during urination and vaginal discharge with different amount and contents Three participants remarked:

"...... everything that I ate come out from my belly looks yellowish, and also I went to the toilet now and then...now and then I had no rest when it became worse, I went to the hospital even though out of earlier 
to my appointment and I got ORS then after some days it stopped and also my itching sensation was different it was day and night it hurt me a lot and subside after I took medicine....".

".... when I was taking treatment, I had burning sensation,

I felt not good in my belly after I ate some food, and I had no appetite....."

And the other one commented on:

"....... My legs got swelled and doctors suspect it might be due to clot formation, he ordered an ultrasound but the radiologist confirmed and suggested there was no any clot formation and that was the effect of Radiation.......".

"....... My problem is sexual intercourse becomes a source of suffering; I would be happy when I could avoid such suffering. My husband and my own difficulty are severing burning sensation if it could avoid my marriage would be saved..........".

Cancer treatment modalities have their own negative impact on women patients with physical, psychological, emotional, and other sexual functional complaints. All participants in this study expressed pains in the intercourse mainly due to less lubricated vagina and add also noticed that vaginal tightening. Another kind of complaint loss of sexual desire due to predetermined decisions to stop sexual intercourse in order to prevent reoccurrence of the disease as they thought. The following comment highlights two women's experiences of their fear of cancer recurrence because of having strong pain after intercourse. Commented on:

"........my pain during sexual intercourse not related to narrowing of my vagina, I think it is due to my cervical cancer not cured... if it was cured, it would not hurt me like this......" (Mother of four children and 40 years old).

"...... I faced for severe burning like pain inside of my vagina during intercourse all the time, and I claim to him to go to down city and satisfy himself freely because day after day the problem has been worsening and become out of my control and I am fearing of the disease may come again......".

\subsection{Delayed effect of treatment}

The late effect of pain symptoms reported by all participants were various types of painful distress symptoms, with back pain being the most common symptoms after internal radiation. The pain intensity could provoke against their daily activities. One commented:

" .........I do not know why such disease on me, sometimes I think it might be as a result of bad works of mine in the past GOD knows everything, I have no any means to know about, my back is just withered I am on duty or at rest through the night whenever the back pain starts the pain is so severe like someone is beaten by an axel on his back then after pain radiated to my womb though I feel pain as if the mother in labor just like that....". 


\subsection{Sexual Problems after radiation therapy}

Cancer treatment modalities have own negative impact on women patients of sexualities. Most women shared their sexual experiences as not willing to have sexual intercourse following cervical cancer treatment due to the disease process.

"I think sexual intercourse is the major source of suffering and thinking the intercourse is impossible to think. The chance of recurrence is also high when you have sex".

"I had experienced fear due to excessive pain during sexual intercourse and didn't have orgasm. So, you don't enjoy intercourse. I hope it might be improved after completion of the treatment"

"....... pain during sexual intercourse not related to narrowing of my vagina, I think it is due to the disease process ...if it is cured it would not hurt me like this......"

\subsection{Knowledge deficient about treatment process and sexual relations}

The third theme that emerged from data analysis was knowledge deficit. Within the theme, one category inadequate information received from the health professionals. According to the study findings, lack of knowledge about the treatment process and sexual relations was reported by most of the interviewed women. Sample responses in this regard included:

"I haven't ever heard about cervical cancer until I was diagnosed with it....... Health providers didn't tell us anything about the treatment process and sexual relations. So, I didn't know whether to have sexual intercourse or not while on treatment".

“.......No one asked me openly about my sexuality. And I am ashamed to talk about the subject [sex]. I didn't feel it is important".

\subsection{Coping strategies of sexual relation}

The study findings reveal that participants held some beliefs about coping strategies of sexual relations with their husbands following cervical cancer treatment. The finding was evident in the following sample responses:

"I don't need to continue sexual intercourse. I just decided it myself to stop even after completion of therapy".

".... / discussed with my husband to stop sexual intercourse due to excessive pain during sexual intercourse and fear of the recurrence of the disease process. After the completion of treatment, we can enjoy it [sex] and has promised me to wait for it...." (A 60 years old mother).

\section{Discussion}


In this study, all participants reported having impaired sexual functioning. The study by Cleary et al. stated that gynecological cancer patients reported having harmful changes in sexual relationships and sexual performance, which experienced sexual dysfunction in every stage of the sexual response [18]. Physical alterations also occurred in cervical cancer patients receiving radiotherapy. Irradiated cervical cancer patient participant reported having decreased vaginal lubrication, loss of sensations, reduced libido, and shortened vagina. These findings were comparable with a study conducted by Rasmussen and Thom that patent with radiotherapy reduced vaginal lubrication and cytostatic treatment, and drugs altered physiological activity (metabolic change) and sexual activities in patient with cervical cancer [19]. And the side effects were unexpected, the patient's sense of control and dignity seemed undermined which may have resulted in feelings of anger and resentment. These results are consistent with previous studies which found that psychological factors such as a sense of control (related to informed decision-making), and self-esteem among others, rather than the nature of the treatment itself, are significant factors in sexual adjustment [20]. A majority of women in this sample felt that they had misconceptions about the treatment and what kind of disease they had and which their expectations based on, which lead to misinterpretations of bodily and functional changes associated with various treatment modalities. Since patients in various stages of shock and distress perceive, understand and recall information differently, the displayed reactions of the patients need not accurately express their inner confusion and thus may mislead the clinician. Therefore, a closer inquiry by the clinician as to how clearly the patient has understood the anatomical and physiological implications of their treatment may prove useful. However, there may well be a case for the introduction of sexual matters at the initial consultations prior to the beginning of treatment schedules, thus laying the foundation for future discussions [21]. It is particularly important that the patient is informed about any potential treatmentinduced changes in her sexual responses. The current results indicated that patients require the services of psychological and medical professionals long after the initial physiological healing is complete. It seems that patients would benefit from discussions specific to sexual matters in follow-up sessions when the recommencement of sexual activity is likely to have occurred [22].

Even though the need for sexual information was found to be very important in cervical cancer patients, most healthcare professionals unsuccessful to address their patients' needs in clinical settings especially sexual related issues because they felt uncomfortable about initiating the discussion or because they had an inadequate time of discussion or knowledge relating to the alterations in sexuality after the diagnosis and treatment of cervical cancer. The patients were become quiet in seeking help because of shame [23]. There is an indication for health care workers to initiate to satisfy patient needs and to implement appropriate timing for patients' helpful psychosocial involvements with their different types of cultural views. Therefore, all health care workers must play great roles to conduct health education regarding women's sexual experiences and adjustment after cervical cancer management.

\section{Limitation of the study}

While results may not be generalizable for the data were collected through Individual Interviews, they may be transferable to other settings of similar features. It should also be clear that the emerged themes were 
substantiated by the local and global works.

\section{Conclusion and Recommendations}

From the analysis of individual interview data, four themes emerged, namely, Treatment Side effect, Sexual Problems after radiation therapy, Knowledge deficiency, and coping strategies of sexual relation. This study is the first of its kind in Ethiopia. The findings of this study added to the existing body of knowledge on the Sexual experience of women after pelvic radiotherapy for cervical cancer in Ethiopia and elsewhere. The sexual lives of women are affected due to various reasons and pelvic radiotherapy for the treatment of cervical cancer. Health professionals should be aware of these effects and should encourage their patients to express their problems and provide effective, individual counsel to each client. The result of this study suggested that there should be a well-established integrated service set up to address the sexual-related problems as a result of treatment side effects on the patient's physical, psychological as well as social problems of the treated women in Tikur Anbessa Specialized Hospital. In addition to that health professionals should accomplish their responsibilities to address the client need, More staffs should be considered to decrease workload and get enough time to tackle the problems which could decrease the influences related to knowledge deficit and the Federal Ministry of Health should give emphasis for women who receive radiotherapy treatment and its consequences on sexual dysfunction and the impact on the quality of life.

\section{Declarations}

\section{Ethics approval and consent to participate}

Ethical approval was obtained from the research and ethical review board of Addis Ababa University, College of health sciences, school of Nursing, and midwifery. All the study subjects provided written informed consent and shared about the purpose and procedure of the study and their right to refuse was respected and consent was obtained from all study participants before the interview. The respondents were also told that the information obtained from them be treated with complete confidentiality and do not cause any harm to them. Methods were carried out in accordance with Helsinki guidelines and regulations.

\section{Consent for publication}

Not applicable.

\section{Availability of data and materials}

Datasets used and/or analyzed during the current study are available from the corresponding author on reasonable request.

\section{Conflict of Interest/ competing interests}


This thesis is submitted in partial fulfilment of the requirement for the MSc degree from the School of Postgraduate Studies at Addis Ababa University, School of Nursing and Midwifery, Oncology Unit. The thesis is deposited in the Library of Addis Ababa University and is made available to the user under the rules of the library. The authors do not have any competing interests.

\section{Funding:}

Funding for this study was made possible through grants offered by the Addis Ababa University postgraduate office.

\section{Authors' Contributions}

The paper is the result of joint research, the contribution of every author is comparable to the others. Gashaw Y. searched the literature, trained field researchers for data collection, and wrote draft results and reviews of the manuscript and Endalew G. has participated in the data analysis, interpretation, and review of the manuscript for publication. Teshome H. Data analysis, Conceptualized the paper(report) and Manuscript preparation and other necessary document preparation for publication. All authors read and approved the final manuscript.

\section{Acknowledgments}

The authors would like to acknowledge to Addis Ababa University, Collage of Health Science, Department of Nursing and the study participant for their unreserved support during data collection.

\section{References}

1. Ferlay J, Soerjomataram I, Ervik M, Dikshit R, Eser S, Mathers C, Rebelo M, Parkin DM, Forman D, Bray F. GLOBOCAN 2012: Estimated Cancer Incidence, Mortality, and Prevalence Worldwide in 2012. International Agency for Research on Cancer. World Health Organization. 20143 [2].

2. Gotay CC, Farley JH, Kawamoto CT, Mearig A. Adaptation and quality of life among long- term cervical cancer survivors in the military health care system. Military medicine. 2008 Oct 1; 173(10):1035-41.

3. Afiyanti Y. Attitudes, Belief, and Barriers of Indonesian Oncology Nurses on Providing Assistance to Overcome Sexuality Problem. Nurse Media Journal of Nursing. 2017 Jul 5; 7(1):15-23.

4. Tigeneh W, Molla A, Abreha A, Assefa M. Pattern of cancer in Tikur Anbessa specialized hospital oncology center in Ethiopia from 1998 to 2010. Int J Cancer Res Mol Mech. 2015; 1(1).

5. Haesler E, Bauer M, Fetherstonhaugh D. Sexuality, sexual health and older people: a systematic review of research on the knowledge and attitudes of health professionals. Nurse education today. 2016 May 1; 40:57-71.

6. Wilmoth MC, Spinelli A. Sexual implications of gynecologic cancer treatments. Journal of Obstetric, Gynecologic, \& Neonatal Nursing. 2000 Jul; 29(4):413-21. 
7. Fernandes WC, Kimura M. Health related quality of life of women with cervical cancer. Latin American nursing journal. 2010 Jun; 18 (3): 360-7.

8. Moelle U, Mathewos A, Aynalem A,Wondemagegnehu T, Yonas B, Begoihn M,Addissie A, Unverzagt S, Jemal A, Thomssen C, Vordermark D. Cervical Cancer in Ethiopia: The Effect of Adherence to Radiotherapy on Survival. The oncologist. 2018 Mar 22: theoncologist-2017.

9. Tigeneh W, Molla A, Abreha A, Assefa M. Pattern of cancer in Tikur Anbessa Specialized Hospital Oncology center in Ethiopia from 1998 to 2010. Int J Cancer Res Mol Mech. 2015;1(1).

10. Bradley EH, Curry LA, Devers KJ. Qualitative data analysis for health services research: developing taxonomy, themes, and theory. Health services research. 2007 Aug;42(4):1758-72

11. Baxter P, Jack S. Qualitative case study methodology: Study design and implementation for novice researchers. The qualitative report. 2008;13(4):544-59)

12. Franklin C, Ballan M. Reliability and validity in qualitative research. The handbook of social work research methods. 2001; 4:273-92.

13. West E. History, organization and the changing culture of care: A historical analysis of the frontier nursing service. Journal of International Women's Studies. 2013; 14(1):21835.

14. Ngwenya D. Research Design, Methodology and Data Collection Methods. InHealing the Wounds of Gukurahundi in Zimbabwe 2018 Springer, Cham (pp. 117-140).

15. Sirajudeen MS, Pillai PS, Shah UN, Mohan N. Content validity and inter-rater reliability of a checklist to assess the ergonomic practice of computer professionals. International Journal of Therapies and Rehabilitation Research. 2012 May 1; 1(2):11.

16. Leonard A, Grobler AF. Exploring challenges to transformational leadership communication about employment equity: Managing organizational change in South Africa. Journal of Communication Management. 2006 Oct 1; 10(4):390-406.

17. Kreuger L, Neuman WL. Social work research methods: qualitative and quantitative approaches: with Research Navigator. Pearson/Allyn and Bacon; 2006

18. Cleary V, Hegarty J, McCarthy G. Sexuality in Irish women with gynecologic cancer. Oncol Nurs Forum 2011; 38: E87- 96

19. Wilmoth MC, Hatmaker-Flanigan E, Laloggia V, Nixon T. Ovarian cancer survivors: Qualitative analysis of the symptom of sexuality. Oncol Nurs Forum 2011; 38: 699-708.

20. Juraskova I, Butow P, Robertson R, Sharpe L, McLeod C, Hacker N. Post-treatment sexual adjustment following cervical and endometrial cancer: A qualitative insight. Psycho-Oncology: Journal of the Psychological, Social and Behavioral Dimensions of Cancer. 2003 Apr; 12(3):267-79.

21. Corney RH, Crowther ME, Everett H, Howells A, Shepherd JH. Psychosexual dysfunction in women with gynaecological cancer following radical pelvic surgery. BJOG: An International Journal of Obstetrics \& Gynaecology. 1993 Jan;100(1):73-8

22. Khoo SB. Impact of cancer on psychosexuality: Cultural perspectives of Asian women. Int J Nurs Pract 2009; 15: 481- 8. 
23. Bukovic D, Silovski H, Silovski T, Hojsak I, Sakle K, Hrgovic Z. Sexual functioning and body image of patients treated for ovarian cancer. Sex Disabil 2008; 26: 63-73. 